\title{
Atlanto-occipital Approach for Cervical Myelography in Horse with Spinal Cord Compression
}

\author{
Cosmin PEȘTEAN*, Liviu OANA, Cristian CRECAN, Alexandra MUREŞAN, Robert PURDOIU, Radu \\ LĂCĂTUŞ, Ciprian OBER
}

Faculty of Veterinary Medicine, University of Agricultural Sciences and Veterinary Medicine Cluj-Napoca, Mănăștur street 3 - 5, Cluj-Napoca, Romania.

*corresponding author: cosmin.pestean@usamvcluj.ro

Bulletin UASVM Veterinary Medicine 75(2)/2018

Print ISSN 1843-5270; Electronic ISSN 1843-5378

doi:10.15835/buasvmcn-vm:001018

\begin{abstract}
The aim of this study was to establish a specific interdisciplinary protocol for evaluation of horses with spinal cord compression. A filly was presented with signs of ataxia at the Faculty of Veterinary Medicine Cluj-Napoca. After neurological examination the presumptive diagnostic was spinal cord compression. Under general anesthesia, the patient was placed for radiological examination in lateral recumbency with head elevated. After antisepsia of cervical region, a Tuohy needle was inserted in atlanto-occipital space and contrast substance was administrated. Radiographic images of the cervical vertebral column were obtained in the neutral, flexed, and extended head positions. The anesthesia protocol was effective, the needle was placed safely in the subarachnoid space and the contrast substance flowed caudally. The obtained radiographic images confirmed spinal cord compressions at the level of cervical vertebrae C3, C4, C5. This working protocol was effective to obtain radiographical images with contrast substance in horses with neurological diseases.
\end{abstract}

Keywords: spinal cord compression, horse, myelography

\section{Introduction}

Incoordination or ataxia in the horse is a common clinical presentation requiring a methodicalapproachforaccurateneurolocalisation (Alcott, 2017). Earlier descriptions of the condition still retain its authenticity: "The power to move with speed is entirely lost, nor is the ability to progress at a slower pace by any means assured" (Mayhew, 1861). There are many causes of ataxia in horses, including equine degenerative myelopathy (Summers et al., 1995) equine protozoal myeloencephalopathy (Summers et al., 1995), equine herpesvirus 1 myeloencephalopathy (Mayhew et al., 1978) rabies, other viral equine encephalomyelidities (Summers et al., 1995), focal cervical injury and trauma, fibrocartilaginous ischemic encephalomyelopathy, and intervertebral disc prolapsed (Summers et al., 1995). Cervical vertebral compressive myelopathy is also known by the names: cervical vertebral stenotic myelopathy (CVSM), cervical vertebral malformation and malarticulation, spinal ataxia, cervical stenotic myelopathy, and "wobbler's" syndrome. It is one of the most common causes of neurologic disease in horses worldwide. At the present time, CVSM seem to be the most appropriate term (Nout and Reed, 2010). Currently, CVSM is separated into 2 forms of spinal cord compression, dynamic or static (Rush Moore et al., 1993).

A presumptive clinical diagnosis is obtained by a combination of a detailed history from the owners or trainers, the subject details of the animal and the recognition of ataxia with neuroanatomical 
localisation caudal to the foramen magnum on neurological examination (Nout and Reed, 2003). Haematology, electromyography and serum biochemistry are generally unremarkable in horses with CVSM. Lateral radiographs of the cervical vertebrae, should reveal bony malformations of the cervical vertebrae. Myelography, a radiographical study of the cervical spinal cord, injecting a contrast agent into the spinal canal. is required to confirm diagnosis of focal spinal cord compression and to identify the location and number of lesions, particularly if surgical treatment is pursued (Neuwirth, 1992).

Taking into account the different possibilities to perform a myelography in equine patients, we wanted to establish a specific interdisciplinary protocol for evaluation of horses with spinal cord compression.

\section{Materials and methods}

A filly of 2,5 years and $320 \mathrm{~kg}$, was presented with signs of ataxia at the Equine Clinic of the Faculty of Veterinary Medicine Cluj-Napoca. Neurological examination was performed to assess symmetry and severity of weakness, ataxia and spasticity. Clinical assessment started with static examination followed by dynamic examination performed walking, circling, elevating the head and maneuvering over obstacles and inclines. Neurologic deficit was graded on a scale of 0 to 5 (Mayhew et al., 1993). Grade 0: Normal strength and coordination. Grade 1: Subtle neurological deficits only noted under special circumstances but mild (e.g. while walking in circles). Grade 2: Mild neurological deficits but apparent atall times/ gaits. Grade 3: Moderate deficits at all times/gaits that are obvious to all observers regardless of expertise. Grade 4: Severe deficits with tendency to buckle, stumble spontaneously, and trip and fall. Grade 5: Recumbent, unable to stand.

Complete blood count and serum chemistry was performed to make informed anesthesia and for excluding other causes for this clinical signs. General anesthesia was performed to make a series of radiographs in neutral, flexed and extended positions of the neck without and with contrast substance (Omnipaque $350 \mathrm{mg} /$ $\mathrm{ml}$ GE Healthcare Norway). For preanesthesia we had used phenothiazines and alfa 2 agonists, induction was performed with benzodiazepines and cyclohexamines. Maintenance of anesthesia was achieved by intravenous bolus injection of alpha 2 agonists and cyclohexamines.

\section{Results and Discussions.}

After clinical and neurological examination, the following clinical aspects were observed: proprioception deficit on the hind legs, hypermetry, vaccilant walking, ataxia which was increasing when walking on a inclined surface and if the horse's head was raised, astasia would occur. This neurological deficit was rated as grade 3 (Moderate deficits at all times/gaits that are obvious to all observers regardless of expertise). The data obtained from the laboratory analyzes have not provided any relevant additional information. After induction of general anesthesia, the horse was placed in lateral recumbency and several radiographs were taken in neutral, flexed and extended positions of the neck.

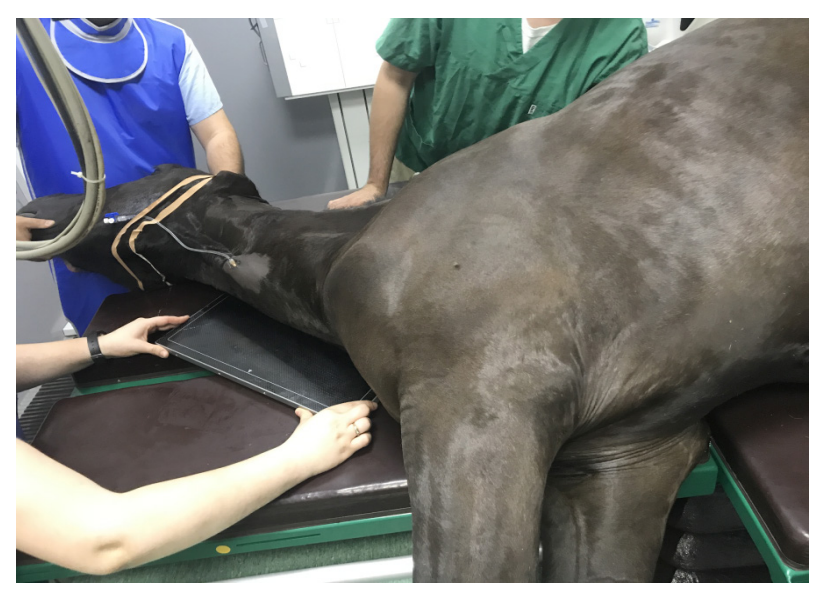

Figure 1 Lateral radiograph of cervical spinal cord in extended position

After thorough antisepsia of cervical region, an 18G Tuohy needle was inserted safely through atlanto-occipital space into the subarachnoid space and cerebrospinal fluid was drained before slowly administrating $50 \mathrm{ml}$ of a nonionic, watersoluble contrast agent.

The head was elevated to an angle of $30^{\circ}$ for 5 to 10 minutes in order to make the radiopaque agent flow caudally. In latero-lateral exposure with the neck in neutral position the contrast medium cover all the subarachnoidean space, highlighting the upper and lower margin of the medullary canal. In the flexed position the ventral margin of the spine present small indentation between 
the C2-C3 and C4-C5 suggesting mild cervical compression.

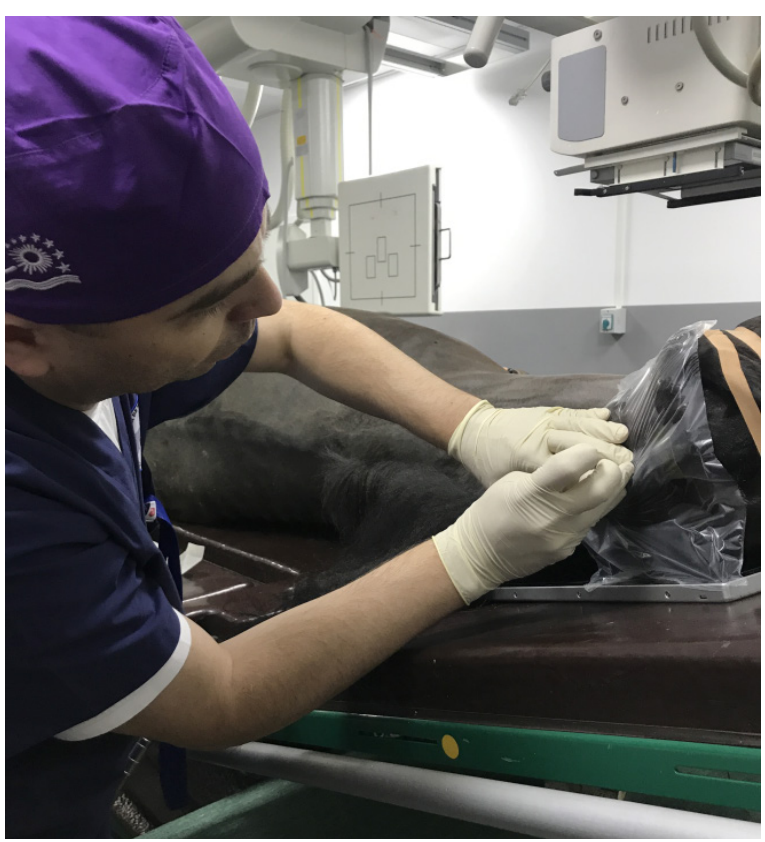

Figure 2 Subarachnoid placement of a Tuohy needle through atlanto-occipital space

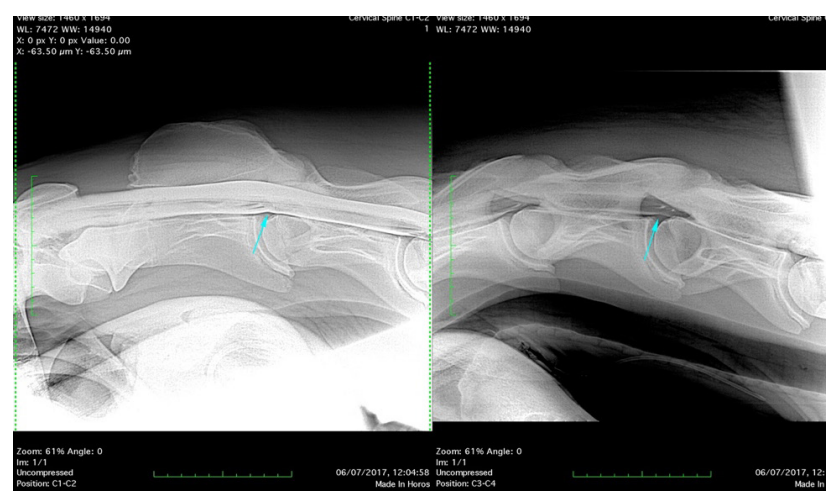

Figure 3 Cervical myelogram presenting mild compression of the spinal cord at C2-C3 and C4-C5

The recovery from anesthesia was not accompanied by unpleasant events, however following general anesthesia and myelography, the clinical signs have worsened, the patient needed constant assistance for getting up and had to be maintained suspended.

\section{Conclusions}

Myelography is regarded as clinically necessary test to demonstrate impingement on the spinal cord by a stenotic vertebral canal, by exuberant periarticular soft tissue or cyst and with or without additional dynamic flexion or extension positioning. The anesthesia protocol was effective, although the entire protocol was quite long. The needle was placed safely in the subarachnoid space and the contrast substance flowed caudally. The analysis of radiographic images with and without contrast substance confirmed the existence of mild spinal cord compressions. We demonstrated the efficiency of this interdisciplinary working protocol to obtain radiographical images with contrast substance in horses with neurological diseases.

Acknowledgments. This research did not receive any specific grant from funding agencies in the public, commercial, or not-for-profit sectors.

\section{References}

1. Alcott CJ (2017). Evaluation of ataxia in the horse. Equine Vet. Educ. Doi: 10.1111/eve.12461

2. Mayhew IG, Donawick WJ, Green SL, Galligan DT, Stanley EK, Osborne J (1993). Diagnosis and prediction of cervical vertebral malformation in Thoroughbred foals based on semiquantitative radiographic indicators. Equine Vet. J., 25: 435-440.

3. Mayhew E (1861). The Illustrated Horse Doctor, D. Appleton and Co., New York, USA.

4. Neuwirth L (1992). Equine myelography. Comp. Cont. Educ. Pract. Vet., 14: 72-78.

5. Nout YS, Reed SM (2003). Cervical vertebral stenotic myelopathy Equine Vet. Educ., 15:212-223.

6. Rush Moore B, Reed SM, Robertson JT (1993). Surgical treatment of cervical stenotic myelopathy in horses: 73 cases (1983-1992). J. Am. vet. med. Ass., 203:108-112.

7. Summers BA, Cummings JF, de Lahunta A (1995). Degenerative diseases of the central nervous system. In:Veterinary neuropathology, (pp. 208-350). Mosby-Year Book, St. Louis, MO.

8. Summers BA, Cummings JF, de Lahunta A (1995). Inflammatory diseases of the central nervous system. Veterinary Neuropathology, (pp. 95-188). Mosby-Year Book, St. Louis, MO.

9. Summers BA, Cummings JF, de Lahunta A (1995). Injuries to the central nervous system. In:Veterinary neuropathology, pp.189-207. Mosby-Year Book, St. Louis, MO. 